\title{
Experience of pain and lifestyle risk factors
}

\author{
Anette West Skaarup ${ }^{1}$, Per Rotbøll Nielsen², Hanne Tønnesen ${ }^{1,3,4}$
}

\begin{abstract}
Background Patients with acute and chronic pain have a significantly reduced quality of life, and it is a clinical impression that unhealthy lifestyle influences the experience of pain. To evaluate this, surgery can be seen as a controlled trauma and acute pain model with standardised guidelines. The aim was to investigate the association between lifestyle risk factors and experience of heavy postoperative pain and their duration after major orthopaedic surgery.

Methods 109 patients undergoing spine, hip or knee surgery were included. Lifestyle was recorded as self-reported alcohol consumption, smoking, physical inactivity, obesity and risk of malnutrition based on the HPH DATA Model. Pain experience was measured on a visual analogue scale of $0-100 \mathrm{~mm}$ for the nociceptive and the less frequent neuropathic pain. Heavy pain was defined as maximal pain $>30 \mathrm{~mm}$ at rest or $50 \mathrm{~mm}$ as dynamic or duration of maximal pain $>40 \mathrm{~min}$. Preoperative pain history and the methods of pain relief were noted. The postoperative pain was followed for three days.

Results Univariate analyses were performed prior to the final multi-variate analyses. Interestingly, unhealthy lifestyle, age or gender were not associated with heavy postoperative pain, except for physical inactivity being negatively associated with the rare neuropathic pain. Unsurprisingly, the study confirmed the significant associations between preoperative and postoperative heavy pain.

Conclusion The results showed no or very little support to the clinical impression that patients with poor lifestyle experience pain different from patients with a healthy lifestyle.
\end{abstract}

\section{About the AUTHOR}

${ }^{1}$ WHO-CC, Clinical Health Promotion Centre, BispebjergFrederiksberg Hospital, University of Copenhagen ${ }^{2}$ Multidisciplinary pain center, Rigshospitalet, University of Copenhagen

${ }^{3}$ Health Science, University of Southern Denmark.

${ }^{4}$ Clinical Health Promotion Centre, Lund University, Sweden.

Contact: Hanne Tønnesen hanne.tonnesen@regionh.dk

\section{Introduction}

Acute and chronic pain is a frequent barrier for a healthy life. Pain significantly reduces the health and quality of life (1), and pain is therefore included in many of the validated scales and questionnaires of health related life quality (2). Chronic pain is often more difficult to relieve than the acute (3). Irreversible plasticity changes of the nerve system after sustained pain are thought to cause the chronic pain, in the more susceptible individuals (4).

Most illnesses and injuries as well as several interventions are followed by pain requiring sufficient treatment. In spite of improved techniques treatment of pain can still today be a challenge, which all physicians, nurses and other health professionals meeting patients as part of their daily work are familiar with.

It is a clinical impression that smokers and hazardous alcohol drinkers experience pain after injuries or surgery different from other patient groups, persons with high level of physical activity can stand more pain without complaining, while obesity is associated with a heavier pain feeling.

This impression of different pain experience for patients with an unhealthy lifestyle may be caused by some extrapolation from the facts that individuals with chronic pain often report using alcohol and cigarettes to cope with the pain $(5 ; 6)$, that chronic pain is reported more frequently among smokers and heavy alcohol drinkers, physical active and obese persons $(6 ; 7)$, and that alcohol abusers may require more anesthesia at surgery (8). However, these do not indicate or explain that the experience of pain should be different for patients with different lifestyles.

In order to investigate the association between lifestyle and experience of pain, postoperative pain would constitute a clinically relevant and standardised model for acute pain. In this model surgery constitutes a very controlled trauma with international validated tools for pain measurement and standardised guidelines. Furthermore, pain after surgery was as stated the most frequent concern among surgical patients (1). 


\section{Research and Best Practice}

The more modern analgesia techniques, such as patient controlled analgesia, peripheral continuous nerve block, and epidural pain analgesia have all proven effective to reduce postoperative pain scores and duration with relative low side effects $(3 ; 9)$. However, so-called breakthrough pain still happens. Reasons for breakthroughs of postoperative pain are perceived to be multi-factorial (10). Predictors of individual pain-thresholds have been investigated (11). Individual factors such as preoperative pain, age, gender, type of surgery, incision size and scores of anxiety are clearly associated with the intensity of pain at the first postoperative hour (11).

Lifestyle may also play a roll - however the association between lifestyle risk factors and prediction of postoperative pain score and pain duration is still unclear (11-13), but evidence shows that improvement of nutrition and physical activity as well as quitting smoking and alcohol can reduce risk of postoperative complications and immobilisation (14-17). Preventing against tthe risk of hese lifestyle factors may also help to improve the postoperative pain relief.

Therefore, we wanted to investigate the association among lifestyle risk factors and experience of heavy postoperative pain and the pain duration after major orthopaedic surgery.

\section{Methods}

The study was a prospective clinical survey with a structured fill-in form for data collection.

\section{Patients}

A number of 109 consecutive, adult patients undergoing major orthopedic surgery at the Head and Orthopedic Centre (HOC), Rigshospitalet, Denmark participated after referral either as elective or acute patients and hospitalized at the orthopedic department one day prior (day -1 ) and one to three days (day +1 to +3 ) after surgery. Preoperative patient characteristics are listed in Table 1.

\section{Pain relieves techniques:}

- Patient-controlled analgesia (PCA) - inclusive postoperative morphine-pumps for epidural or intravenous administration (18),

- Regional analgesia (RA) - inclusive postoperative pumps for epidural administration (18) and peripheral nerve blocks

- Conventional analgesia (CA) - inclusive oral administration of primary and eventually secondary analgesics.

All three groups could have had the possibility ofpro re nata $(\mathrm{pn})$ intravenous bolus-injections of opioid.
Table 1 Patient characteristics, given in numbers (\%) or median (range)

$\begin{array}{lrrr}\text { Demographic } & \begin{array}{r}\text { Woman } \\ \mathbf{n}=\mathbf{5 7}\end{array} & \begin{array}{r}\text { Men } \\ \mathbf{n}=\mathbf{5 2}\end{array} & \begin{array}{r}\text { Total } \\ \mathbf{n}=\mathbf{1 0 9}\end{array} \\ \text { Gender } & 52 \% & 48 \% & 100 \% \\ \text { Age, year } & 62(20-85) & 60(19-83) & 60(19-85)\end{array}$

Type of referrals and classification of operation

Acute + elective referrals

$18 \%+82 \% \quad 27 \%+73 \% \quad 22 \%+78 \%$

Column spine

Knee

$32 \% \quad 25 \% \quad 28 \%$

$28 \% \quad 27 \% \quad 28 \%$

Hip

Others (pelvic, shoulder)

$25 \% \quad 23 \% \quad 24 \%$

Fractures

$10 \%$

$2 \%$

$6 \%$

Preoperative heavy pain

Duration $>2$ months

$5 \% \quad 23 \% \quad 14 \%$

VAS $_{\text {rest }}>30$, nociceptive + neuropathic

$\begin{array}{rrr}88 \% & 75 \% & 82 \% \\ 26 \%+4 \% & 15 \%+12 \% & 21 \%+7 \% \\ 27 \%+7 \% & 25 \%+10 \% & 26 \%+9 \%\end{array}$

VAS $_{\text {dyn }}>50$, nociceptive + neuropathic

$27 \%+7 \% \quad 25 \%+10 \% \quad 26 \%+9 \%$

$(n=104)$

Durationa*, nociceptive + neuropathic $36 \%+11 \% \quad 15 \%+6 \% \quad 26 \%+8 \%$ $(n=108)$

Taking pain medication

$90 \% \quad 75 \%$

$83 \%$

\section{Lifestyle risk}

\begin{tabular}{|c|c|c|c|}
\hline Risk of malnutrition ${ }^{b^{*}}$ & $35 \%$ & $29 \%$ & $32 \%$ \\
\hline Body Mass Index $<20.5 \mathrm{~kg} / \mathrm{h} 2$ & $14 \%$ & $6 \%$ & $9 \%$ \\
\hline \multicolumn{4}{|l|}{ Body Mass Index $<20.5$} \\
\hline median & 19.0 & 19.7 & 19.6 \\
\hline range & $(16.9-20.5)$ & $(19.6-19.8)$ & $(16.9-20.5)$ \\
\hline Weight loss last 3 months & $14 \%$ & $15 \%$ & $15 \%$ \\
\hline Decreased calorie-intake last 7 days & $16 \%$ & $19 \%$ & $17 \%$ \\
\hline Stress metabolic, 3rd degree & 0 & 0 & 0 \\
\hline Overweight $^{\mathrm{b}^{*}}$ & $74 \%$ & $64 \%$ & $69 \%$ \\
\hline Body Mass Index $>25$ & $58 \%$ & $56 \%$ & $57 \%$ \\
\hline \multicolumn{4}{|l|}{ Body Mass Index $>25 \mathrm{~kg} / \mathrm{h}^{2}$} \\
\hline median & 26.6 & 29.0 & 27.4 \\
\hline range & $(25.1-39.1)$ & $(25.1-54.1)$ & $(25.1-54.1)$ \\
\hline Waist: $W>80 \mathrm{~cm} / M>94(n=106)$ & $72 \%$ & $62 \%$ & $67 \%$ \\
\hline \multicolumn{4}{|l|}{ Waist: $W>80 \mathrm{~cm} / \mathrm{M}>94$} \\
\hline median & 94.5 & 108 & 100 \\
\hline range & $82-126$ & $95-148$ & $82-148$ \\
\hline $\begin{array}{l}\text { Physical activity the last month }<30 \\
\text { min daily }\end{array}$ & $70 \%$ & $54 \%$ & $62 \%$ \\
\hline Smoking daily & $18 \%$ & $27 \%$ & $22 \%$ \\
\hline Cigarettes daily & $19(3-20)$ & $15(2-30)$ & $16(2-30)$ \\
\hline $\begin{array}{l}\text { Risky alcohol, } W>14 \text { / M >21 drinks } \\
\text { weekly }\end{array}$ & $3(5.3 \%)$ & $7(13.5 \%)$ & $10(9.2 \%)$ \\
\hline Daily alcohol intake by risky drinkers & $42(27-53)$ & $35(25-70)$ & $35(25-70)$ \\
\hline
\end{tabular}

a* VAS $_{\text {max }}>50$ for $>40$ minutes; $\quad$ b* Defined as any positive symptom below; 


\section{Research and Best Practice}

\section{Inclusion criteria}

Major orthopedic surgery of fractures or degenerative etiology, such as knee, hip, and shoulder prostheses or prostheses revision, femoral marrow pin, spinal column and pelvic operations.

\section{Exclusion criteria}

Patients under 18 years, reduced competence or unwillingness to give informed consent, inability to understand Danish, operations due to tumor or metastases, or preoperative lack of pain sensation in the operation field.

\section{Outcomes}

Lifestyle

The Health Promoting Hospital (HPH) DATA Model for risky health among patients was used (19). According to the clinical routines, patients self-reported their lifestyle except for the waist circumference, which was measured by the project personnel.

Five lifestyle related risk factor meassurements:

- Risk of malnutrition if at least one of the following: $\mathrm{BMI}<20.5 \mathrm{~kg} / \mathrm{m} 2$, weight loss during last 3 months, eaten less last week or patient stress metabolic in a $3^{\text {rd }}$ degree.

- Overweight if at least one of the following: BMI > $25 \mathrm{~kg} / \mathrm{m} 2$ or waist circumference $>80 \mathrm{~cm}$ for the women and $>94 \mathrm{~cm}$ for the men.

- Physical inactivity: less than 30 minutes activity daily with pulse increase during the last month.

- $\quad$ Smoking: daily smoking

- High alcohol intake: weekly intake of drinks of alcohol $>14$ objects for women or $>21$ objects for men, please

\section{Heavy Pain quantification}

We used a patient-generated visual analogue scale (VAS), from o to 100, given in millimetres $(\mathrm{omm}=$ no pain, $30 \mathrm{~mm}=$ modest pain, $50 \mathrm{~mm}=$ modest-severe pain and $100 \mathrm{~mm}=$ worst imaginable pain). We measured worst pain $\left(\mathrm{VAS}_{\text {max }}\right)$, least pain $\left(\mathrm{VAS}_{\text {min }}\right)$, average pain $\left(\mathrm{VAS}_{\text {mean }}\right)$, rest pain $\left(\mathrm{VAS}_{\text {rest }}\right)$ and pain at mobilization $\left(\mathrm{VAS}_{\mathrm{dyn}}\right.$ ). 'Rest' was defined as lying in bed and 'dynamic' defined as sitting at bedside or standing up, but if patients were immobilized then pain during coughing was accepted. We used the local and clinical relevant definition of heavy pain with three separate outcomes: $\mathrm{VAS}_{\text {rest }}>30 \mathrm{~mm}$ or $\mathrm{VAS}_{\mathrm{dyn}}>50 \mathrm{~mm}$ or daily duration of VAS $>50 \mathrm{~mm}$ for $>40$ minutes.

\section{Type of pain}

The two main types were characterized by their different pathophysiology and treatment; nociceptive pain as cutting, dull, squeezing, cramp-like and easy to locate pain; neuropathic pain as stabbing neuralgias (lancinating, electrifying) or superficial change in sensation (18).

\section{Time}

The duration of breakthrough-pain was measured in seconds, minutes or hours and the breakthroughs in numbers per day. The duration equalled the self-rated average duration of a single pain breakthrough on a particular day. The total time the patient experienced pain was measured as (average duration of a single pain breakthrough) x (number of breakthroughs).

The mobilization time was estimated by the patients and given in hours from o to 12 or more than 12 hours daily.

\section{Ethical considerations}

The patients participated after informed consent. The Danish Data Protection Agency approved the study before start (No 2009-41-3759). There were no risks by participating, but inconvenience from the time spent to report the daily pain and possibly discomfort from mobilization once daily. This survey did not involve human biological material or evaluation of medication, surgical or anaesthesiological equipments, and should therefore not be evaluated by the Scientific Ethical Committee according to the Danish Guidelines for Research following the Helsinki-II Declaration (20). Eligible patients were informed orally and in writing about the purpose and implication of the study. Orally informed consent was obtained before inclusion. Patients could withdraw their consent at any time, without any explanation or implication for their further treatment.

\section{Data-collection}

The fill-in form used for data collection was based on the questionnaires described in previous studies $(16 ; 19)$. Data collection on pain-duration was added. A one-week pilot test was performed successfully, and the case report form was established. All data were collected by project personnel. Eligible patients were identified via the operation-plans for the orthopedic surgeries by a daily manual search. Data on pain and lifestyle were retrieved from patients orally. The data on postoperative diagnosis, operation code and pain-relieve medication were retrieved from the hospital data system.

Day -1, preoperatively: Identification of eligible patients fulfilling the inclusion but not the exclusion criteria - followed by obtaining of informed consent. Data assessed were pain scores, duration of pain breakthroughs, duration of mobilization daily, extension of preoperative pain (under or above 2 months), pain relief medication and lifestyle.

Day o: Day of surgery. 


\section{Research and Best Practice}

Day +1 to +3 , postoperatively: Diagnosis and operation code were registered. Time of discharge from the recovery room was used as starting point for the postoperative pain-relief registration. Every day data collected at bedside on pain severity, duration and number of pain breakthroughs and duration of mobilization were assessed. Postoperative pain medication was registered.

\section{Data analyses}

A percentage of $85 \%$ of the included patients were expected to have one or more clinical relevant lifestyle risk factors (19). About 100 patients were perceived to be relevant for the statistical regression analysis including about 10 variables.

The results were given in medians and ranges. Uni- and multivariate analyses were used for associations between postoperative pain relief and lifestyle. The confounder control included age group (19-49, 50-69 and $70+$ ), gender, operation procedure (grouped in spine column, knee, hip, others, fractures), referral (acute/ elective), preoperative pain level $\left(\mathrm{VAS}_{\mathrm{rest}}>30 \mathrm{~mm}, \mathrm{VAS}_{\mathrm{dyn}}\right.$ $>50 \mathrm{~mm}$ ) and analgesic technique (PCA, RA or CA).

For the univariate analyses, the Chi-square-test with a level of significance at $\mathrm{p}<0.05$ was used.

In the multivariate analyses associations between primary outcome VAS $_{\text {rest }}>30 \mathrm{~mm}, \mathrm{VAS}_{\mathrm{dyn}}>50 \mathrm{~mm}$ and duration of $\mathrm{VAS}_{\text {max }}>50 \mathrm{~mm}>40$ minutes) and any of the five lifestyle risks (malnutrition, overweight, physical inactivity, smoking and high alcohol intake) were tested in combination with significant variables from the univariate analyses. Backwards selection was used. The final results were presented in odds ratio (OR) with 95\% confidence interval (CI). CI not including the value 1 was considered significant. The statistics were performed using SPSS 18.0.

\section{Results}

In total 142 relevant major orthopedic, non-cancer operations with eligible adult patients were identified. Out of those 33 patients were excluded: 3 refused to participate, 4 withdrew their informed consent, 21 had the operation date cancelled and another 5 had language barriers, were multi-traumatized or not available in the department (figure 1).

The characteristics showed that the majority of these orthopaedic patients had suffered from preoperative diagnose-related pain for more than 2 months. The prevalence of risky lifestyle was high. Overweight and physical inactivity were the most frequent unhealthy lifestyles with mostly women at risk. Smoking was the least frequent lifestyle risk factor (table 1). The distribution of

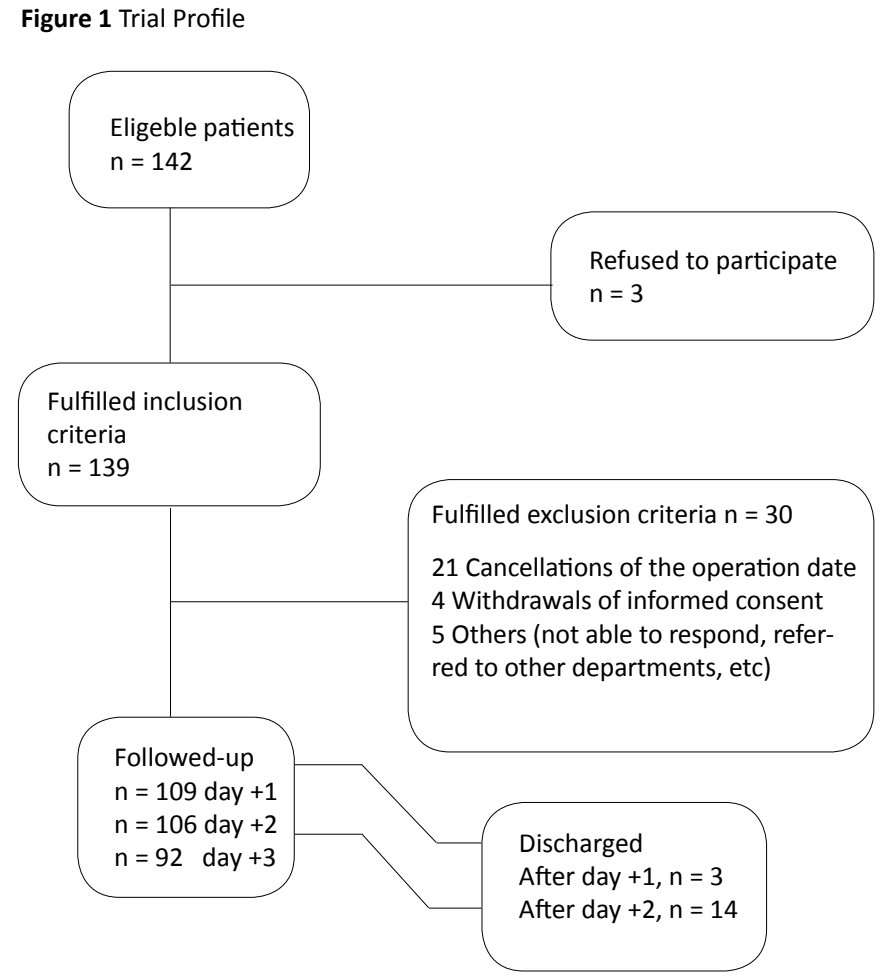

postoperative analgesia technique was RA for about half of the patients, while only $17 \%$ used PCA.

A relative high number of patients exceeded the limits for heavy pain on the first postoperative day, the large majority being the nociceptive type (table 2 ).

The significant results from the univariate analyses have been reported for each outcome in table 3. The final multivariate analyses identified several associations between preoperative and postoperative heavy pain - both for the nociceptive and neuropathic type. Interestingly, unhealthy lifestyle, age or gender were not associated with heavy postoperative pain, except for physical inactivity being negatively associated with neuropathic pain.

\section{Discussion}

This study found almost no associations between lifestyle and experiencing postoperative pain. Only preoperative physical inactivity was associated with less neuropathic postoperative pain and postoperative pain duration. Non-surprisingly, the study confirmed that heavy preoperative pain was significantly associated with experience of heavy postoperative pain, and that the majority of pain constituted of the nociceptive type $(4 ; 11)$. The decline of nociceptive pain with the postoperative time has also been reported before (9). 


\section{Research and Best Practice}

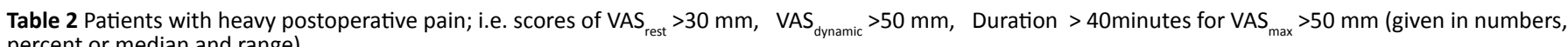
percent or median and range).

VAS: Visual analogue scale 1-100mm; noci: nociceptive pain; neuro: neuropathic pain

Day +1

Day +2

Day +3

\begin{tabular}{|c|c|c|c|c|c|c|c|c|c|}
\hline & $\mathbf{n}$ & Pain score & Heavy pain (\%) & $\mathbf{n}$ & Pain score & Heavy pain (\%) & $\mathbf{n}$ & Pain score & Heavy pain (\%) \\
\hline VAS $_{\text {rest } / \text { noci }}$ & 109 & $20(0-100)$ & $39 \%$ & 106 & $10(0-100)$ & $22 \%$ & 92 & $10(0-100)$ & $22 \%$ \\
\hline VAS $_{\text {rest /neuro }}$ & 108 & $0(0-100)$ & $7 \%$ & 105 & $0(0-80)$ & $6 \%$ & 92 & $0(0-58)$ & $11 \%$ \\
\hline VAS $_{\text {dyn } / \text { noci }}$ & 104 & $49(0-100)$ & $46 \%$ & 103 & $35(0-100)$ & $33 \%$ & 91 & $25(0-100)$ & $15 \%$ \\
\hline $\operatorname{VAS}_{\mathrm{dyn} / \text { neuro }}$ & 104 & $0(0-100)$ & $3 \%$ & 102 & $0(0-80)$ & $5 \%$ & 90 & $0(0-87)$ & $4 \%$ \\
\hline Duration $_{\text {-noci }}$ & 106 & $20(0-1515)$ & $31 \%$ & 104 & $10(0-1470)$ & $23 \%$ & 91 & $15(0-1730)$ & $17 \%$ \\
\hline Duration $_{\text {-neuro }}$ & 107 & $0(0-1110)$ & $1 \%$ & 103 & $0(0-1430)$ & $6 \%$ & 91 & $0(0-1620)$ & $2 \%$ \\
\hline
\end{tabular}

Table 3 Significant associations between preoperative variables and heavy postoperative pain (i.e. scores of VAS $>30 \mathrm{~mm}, \mathrm{VAS}_{\text {dynamic }}>50 \mathrm{~mm}$ Duration $>40$ min for VAS $>50 \mathrm{~mm}$ ) from uni- and multivariate analyses, given in p-values and OR with $95 \% \mathrm{Cl}$. VAS: Visual analogue scale 1-100mm; noci: nociceptive pain; neuro: neuropathic pain; NS: not significant.

\begin{tabular}{|c|c|c|c|c|c|c|}
\hline \multirow[t]{2}{*}{ Nociceptive pain } & \multicolumn{3}{|c|}{ Univariate analyses: $p$-values } & \multicolumn{3}{|c|}{ Multi-variate analyses: OR (95\%-C.I.) } \\
\hline & VAS $_{\text {rest }}>30 \mathrm{~mm}$ & $\mathrm{VAS}_{\text {dynamic }}>50 \mathrm{~mm}$ & Duration & VAS $_{\text {rest }}>30 \mathrm{~mm}$ & $\mathrm{VAS}_{\text {dynamic }}>50 \mathrm{~mm}$ & Duration \\
\hline \multicolumn{7}{|c|}{ Heavy preoperative pain } \\
\hline Pain Treatment & 0.030 & NS & NS & - & & \\
\hline $\mathrm{VAS}_{\text {rest } / \mathrm{noci}}>30 \mathrm{~mm}$ & $<0.000$ & 0.0040 & 0.002 & - & $5.18(1.59-16.86)$ & $5.06(1.29-19.90)$ \\
\hline $\mathrm{VAS}_{\text {rest } / \text { neuro }}>30 \mathrm{~mm}$ & 0.014 & 0.014 & NS & $10.28(1.12-94.46)$ & - & \\
\hline $\mathrm{VAS}_{\text {dyn } / \text { noci }}>50 \mathrm{~mm}$ & 0.003 & $<0.000$ & 0.001 & $4.30(1.60-11.60)$ & - & $4.75(1.53-14.73)$ \\
\hline VAS $_{\text {dyn } / \text { neuro }}>50 \mathrm{~mm}$ & 0.018 & 0.029 & NS & - & - & \\
\hline \multicolumn{7}{|l|}{ Preoperative lifestyle } \\
\hline Malnutrition Risk & NS & NS & NS & & & \\
\hline Overweight & NS & NS & NS & & & \\
\hline Physical inavtivity & NS & NS & NS & & & \\
\hline Smoking & NS & NS & NS & & & \\
\hline Risky alcohol intake & NS & NS & NS & & & \\
\hline \multicolumn{7}{|c|}{ Demographic variables } \\
\hline Gender & NS & NS & NS & & & \\
\hline Age & NS & NS & NS & & & \\
\hline
\end{tabular}

\begin{tabular}{llll} 
Neuropathic pain & \multicolumn{2}{c}{ Univariate analyses: $p$-values } & Multi-variate analyses: OR (95\%-C.I.) \\
& $\mathrm{VAS}_{\text {rest }}>30 \mathrm{~mm} \quad \mathrm{VAS}_{\text {dynamic }}>50 \mathrm{~mm} \quad$ Duration & $\mathrm{VAS}_{\text {rest }}>30 \mathrm{~mm} \quad \mathrm{VAS}_{\text {dynamic }}>50 \mathrm{~mm} \quad$ Duration
\end{tabular}

\section{Heavy preoperative pain}

\begin{tabular}{|c|c|c|c|c|c|c|}
\hline Pain Treatment & NS & NS & NS & & & \\
\hline $\mathrm{VAS}_{\text {rest } / \text { noci }}>30 \mathrm{~mm}$ & NS & 0.031 & NS & & - & \\
\hline $\mathrm{VAS}_{\text {rest } / \mathrm{neuro}}>30 \mathrm{~mm}$ & $<0.000$ & 0.001 & NS & - & - & \\
\hline VAS $_{\text {dyn } / \text { noci }}>50 \mathrm{~mm}$ & $<0.000$ & NS & 0.007 & $10.42(2.36-46.01)$ & & $68.8(3.31-1430.59)$ \\
\hline $\mathrm{VAS}_{\mathrm{dyn} / \text { neuro }}>50 \mathrm{~mm}$ & NS & $<0.000$ & NS & & 17.39 (2.70-111.82) & \\
\hline \multicolumn{7}{|l|}{ Preoperative lifestyle } \\
\hline Malnutrition Risk & NS & NS & NS & & & \\
\hline Overweight & NS & NS & NS & & & \\
\hline Physical inavtivity & NS & 0.043 & 0.028 & & $0.17(0.03-0.95)$ & $0.08(0.008-0.76)$ \\
\hline Smoking & NS & NS & NS & & & \\
\hline Risky alcohol intake & NS & NS & NS & & & \\
\hline \multicolumn{7}{|c|}{ Demographic variables } \\
\hline Gender & 0.032 & NS & NS & - & & \\
\hline Age & NS & NS & NS & & & \\
\hline
\end{tabular}




\section{Research and Best Practice}

\section{Pain after surgery}

Development of postoperative pain is related to the type of surgery, incision size and preoperative pain as well as age and anxiety $(4 ; 11)$. We identified about one third of the patients having heavy pain at rest, increasing to half of patients during mobilisation on the first day after the major surgery. This exceeded our local recommendations and the higher international limits for good quality recommended over ten years ago by the United Kingdom Audit Commission (21). However, it has been commented that pain breakthroughs are unavoidable in the postoperative period $(3 ; 22)$.

The close association of postoperative pain to this type of surgery has been described in a previous Danish hospital study on individual pain-recordings. The prevalence of heavy postoperative pain across surgical interventions was lower, about $15 \%$, compared to $55-78 \%$ in the patient group similar to ours undergoing hip surgery, joint replacement or spine surgery (22). The definition of heavy pain was different; above VAS 30 one or more time during the first postoperative period, but we found similar results when using the same definition; $42 \%$ for nociceptive and $15 \%$ for neuropathic pain.

The duration of pain is seldom included in pain studies, even though it would be an important parameter for the patients in addition to the intensity of pain. Nevertheless, Bardiau and colleagues measured the duration of the total $\mathrm{VAS}_{\max }$-periods and the total time for VASscores above thirty (23). Those figures are not directly comparable, but give the impression that pain breakthroughs for $\mathrm{VAS}_{\max }$ are ongoing in one tenth of the total postoperative time. A small pilot study has found that $70 \%$ of patients refused analgesics when offered, thereby accepting otherwise treatable pain. The same study also showed that another $23 \%$ were not offered analgesics, at all (23). If the pilot results could be translated to our study, they indicate a need for improved health literacy among both patients and staff concerning pain services.

\section{Preoperative lifestyles and postoperative pains}

It is interesting that we could not identify associations between heavy pain and unhealthy lifestyles, except for physical inactivity and less heavy pain. This is in contrast to the well-established associations with development of postoperative complications (24-27) and the strong evidence for effect of intensive lifestyle interventions starting prior to surgery (14-17). Similar evidence does not exist for postoperative pain. Association between smoking and postoperative pain had neither been possible to find for two other studies on minor surgical interventions on knee and tooth, where the postoperative pain level may have been too low to show any association relation pain
$(12 ; 13)$. The Quetelet-index (indicator of body mass) was neither found as an individual predictor of postoperative pain in the study by Kalkman et al (11).

Surprisingly, we found a significant association of preoperative physical inactivity and neuropathic pain and pain duration. It is known that neuropathic pain shows abnormal summation and post-sensations of pain after stimulation (18). In case less physical activity would give less stimulation of the damage pain leading system, maybe this could explain the association.

\section{Study bias and limitations}

Several biases were introduced in our study. The study design introduced a recall bias by using self-reported data, thereby leading to risk of both over-and underestimation. We failed to show associations with age, gender or type of surgery to the postoperative pain as in previous studies (11). It could be due to the relative small size of our study with the risk of lack of power and type-2 failure. Missing data from the third postoperative day may also have played a role for the association between lifestyle and postoperative pain by overlooking a possible late association.

The association between physical inactivity and lower postoperative neuropathic pain could be a type- 1 failure caused by chance from the several analyses performed. In order to reduce the risk of type- 1 failures we have included only the significant results with p-values of 0.05 and lower from the univariate analyses into the multivariate analysis. This is in contrast to other studies allowing p-values of 0.15 (11). We chose not to lower the level of significance, because the variables tested were perceived partly dependent by logic and from the literature (e.g. VAS scores of minimal, maximal and mean pain from the same patient, overweight and physical inactivity, operation procedure and postoperative pain relief, men and their alcohol intake, smoking and alcohol intake). The dependency minimized the risks of masssignificance and the requirement for Bonferroni Correction (28). Though it is relevant from a clinical treatment perspective to divide pain into nociceptive and neuropathic types it seemed to be rather unique for our study. Therefore, the patients could have found it difficult to describe the pain type experienced, while repeated measurements could have improved the reliability.

It is a strength that the study is performed in real life on a patient group often developing heavy pain after surgery and that only few patients refused to participate, which facilitate the translation to other departments with similar patient groups and similar use of pain relief. It also strengthening the study that we confirmed the well- 


\section{Research and Best Practice}

established association to preoperative pain previously published.

\section{Study perspectives}

This study could not accept the hypothesis that postoperative heavy pain is associated with postoperative pain. Thus, it did not support the clinical impression that an unhealthy lifestyle would influence the experience of postoperative pain. Only physical inactivity was associated with reduced neuropathic pain, which was however a rather seldom type of pain compared to the more usual nociceptive pain after surgery. This lonely association should be interpreted with caution, because of the very low number of patients with neuropathic pain in our study, also reflected in the rather wide confidence interval of the odds ratio. The association should be reevaluated in a larger study with patients more frequently developing neuropathic pain after surgery.

\section{Conclusion}

The results showed no or very little support to the clinical impression that patients with poor lifestyle experience pain different from patients with a healthy lifestyle.

\section{Contribution Details}

All authors have drafted, disucssed and revised the final manuscript. All authors designed the study, analyzed and interpreted the results. All authors approved the final version of the manuscript.

\section{Competing Interests}

None declared.

\section{References}

(1) Apfelbaum JL, Chen C, Meilpa SM, et al. Postoperative pain experience: results from a national survey suggest postoperative pain continues to be under managed. Anesth Analg 2003; 97:534-40.

(2) Bowden A1, Fox-Rushby JA. A systematic and critical review of the process of translation and adaptation of generic health-related quality of life measures in Africa, Asia, Eastern Europe, the Middle East, South America. Soc Sci Med 2003; 57:1289-306.

(3) Dolin SJ, Chasman JN, Bland JM. Effectiveness of acute postoperative pain management: I. Evidence from published data. Br J Anaesth 2002; 89:409-23. (4) Rotbøll-Nielsen P, Rudin Å, Werner MU. Prediction of postoperative pain. Curr Anaesth Crit Care 2007; 18:157-65.

(5) Chapman SL, Wu LT. Associations between cigarette smoking and pain among veterans. Epidemiol Rev 2015; 37:86-102. doi: 10.1093

(6) Lübbeke A, Moons KG, Garavaglia et al. P. Outcomes of obese and nonobese patients undergoing revision total hip arthroplasty. Arthritis Rheum 2008; 59:738-45.

(7) Schmelzer AC1, Salt E, Wiggins A, Crofford LJ, Bush H, Mannino DM. Role of Stress and Smoking as Modifiable Risk Factors for Non-persistent and Persistent Back Pain in Women. Clin J Pain 2015 Apr 15. [Epub ahead of print]

(8) Moran S, Isa J, Steinemann S Perioperative management in the patient with substance abuse. Surg Clin North Am. 2015;95:417-28.

(9) Pöpping DM, Zahn PK, Van Aken HK, et al. Effectiveness and safety of postoperative pain management: a survey of 18,925 consecutive patients between 1998 and 2006 (2nd revision): a database analysis of prospectively raised data. Br J Anaesth 2008;101:832-40.

(10) White PF, Kehlet $\mathrm{H}$. Improving postoperative pain management. Anesthesiology 2010; 112:220-5.

(11) Kalkman CJ, Visser K, Moen J, et al. Preoperative prediction of severe postoperative pain. Pain 2003; 105:415-23.

(12) Chimbira W, Sweeney BP. The effect of smoking on postoperative nausea and vomiting. Anaesthesia 2000; 55:540-4.

(13) Lopez-Carriches C, Gomez-Font R, Martinez-Gonzalez JM, et al. Influence of smoking upon the postoperative course of lower third molar surgery. Med Oral Patol Oral Cir Bucal 2006; 11:56-60.

(14) Thomsen T, Villebro N, Møller AM. Interventions for preoperative smoking cessation. Cochrane Database Syst Rev. 2014 Mar 27;3:CD002294.

(15) Oppedal K, Møller AM, Pedersen B, Tønnesen H. Preoperative alcohol cessation prior to elective surgery. Cochrane Database Syst Rev. 2012 Jul 11;7:CD008343.

(16) Rotbøll-Nielsen P, Jørgensen LD, Dahl B, Tønnesen H. Prehabilitation and early rehabilitation after spinal surgery: randomized clinical trial. Clin Rehabbli 2010; 24:137-48

(17) Burden S, Todd C, Hill J, Lal S. Pre-operative nutrition support in patients undergoing gastrointestinal surgery. Cochrane Database Syst Rev. 2012 Nov 14;11:CD008879

(18) Nielsen PR. Interdisciplinary health promotion and rehabilitation program at surgery. Clin Health Promot 2014; 4: Suppl 3 (English abstract 7-8).

(19) Tønnesen $H$, Svane JK, Lenzi L et al. Handling clinical health promotion in the HPH DATA Model: Basic documentation of health determinants in medical records of tobacco, malnutrition, overweight, physical inactivity and alcohol. Clin Health Promot 2012; 2:5-11

(20) www.etiskraad.dk with link to www.retsinformation.dk/Forms/R0710. aspx?id=29142, § 7.1

(21) Audit Commission. Anaesthesia under examination: The efficiency and effectivness of anaesthesia and pain relief services in England and Wales. London: Audit Commission; 1997.

(22) Shapiro A, Zohar E, Kantor M, Memrod J, et al. Establishing a nursebased, anesthesiologist-superviced inpatient APS: experience of 4.617 patients. J Clin Anesth

2004; 6:415-20.

(23) Bardiau FM, Taviaux NF, Albert A, et al. An intervention study to enhance postoperative pain management. Anesth Analg 2002; 96:179-85.

(24) Carr EC, Refusing analgesics: using continuous improvement to improve pain management on a surgical ward. J Clin Nurs 2002; 11:743-52.

(25) Sadr Azodi O, Adami J, Lindström D, Eriksson KO, Wladis A, Bellocco R High body mass index is associated with increased risk of implant dislocation following primary total hip replacement: 2,106 patients followed for up to 8 years.Acta Orthop. 2008; 79:141-7.

(26) Eliasen M, Grønkjær M, Skov-Ettrup LS, Mikkelsen SS, Becker U, Tolstrup JS, Flensborg-Madsen T. Preoperative alcohol consumption and postoperative complications: a systematic review and meta-analysis. Ann Surg. 2013; 258:930-42

(27) Grønkjær M, Eliasen M, Skov-Ettrup LS, et.al. Preoperative smoking status and postoperative complications: a systematic review and meta-analysis. Ann Surg. 2014; 259:52-71

(28) Onerup A, Angerås $U$, Bock D, et.al. The preoperative level of physical activity is associated to the postoperative recovery after elective cholecystectomy - A cohort study. Int J Surg 2015; 19:35-41.

(29) Armstrong RA. When to use the Bonferroni correction. Ophthalmic Physiol Opt. 2014; 34:502-8. 